\title{
Towards Human-Centred Design
}

\author{
Liam J. Bannon \\ Interaction Design Centre \\ Dept. of Computer Science \& information Systems \\ University of Limerick \\ Ireland \\ liam.bannon@ul.ie
}

\begin{abstract}
The field of HCI has evolved and expanded dramatically since its origin in the early 1980's. The HCI community embraces a large community of researchers and practitioners around the world, from a variety of disciplinary backgrounds in the human and social sciences, engineering and informatics, and more recently, the arts and design disciplines. This kaleidoscope of cultures and disciplines as seen at INTERACT Conferences provides a rich pool of resources for examining our field. Applications are increasingly exploring our full range of sensory modalities, and merging the digital and physical worlds. WiFi has opened up a huge design space for mobile applications. A focus on usability of products and services has been complemented by an emphasis on engagement, enjoyment and experience. With the advent of ubiquitous computing, and the emergence of "The Internet of Things", new kinds of more open infrastructures make possible radically new kinds of applications. The sources of innovation have also broadened, to include human and social actors outside of the computing and design organizations. The question is to what extent is our mainstream thinking in the HCI field ready for the challenges of this Brave New World? Do the technological and social innovations that we see emerging require us to re-shape, or even, re-create, our field, or is it a case of a more gradual evolution and development of that which we already know? In this closing Keynote, I will provide a perspective on the evolution and development of the HCI field, looking backwards as well as forwards, in order to determine what are some of the changes of significance in the field. This "broad-brush" approach to what I term " human-centred design" will be complemented by the examination of specific projects and applications, to help anchor some of the discussion. Areas such as user-centred design, participatory design, computersupported cooperative work and learning, and interaction design, in which I have had some involvement over the years, will be mentioned. I will discuss the themes of "ecologies of artefacts", appropriation, tinkering/bricolage, and the emergence of design anthropology, among other topics. The purpose of the talk is not to engage in a form of Futurism concerning the HCI field, but to examine some of the technical and social trends that can be observed, and to highlight some areas of particular significance that warrant further attention. I argue for a multi-layered approach that, while exploring new avenues of research concerning people's use of technology, does not necessarily dismiss the corpus of knowledge we have built up over the years concerning human-computer interaction. From a personal perspective, issues such as means and ends, our
\end{abstract}


underlying values, and concern for our fellow human beings in an increasingly fragile world, are issues that, while perhaps seen as outside the remit of a narrow HCI brief, impact on the field in significant ways. In this regard, discussions of our future should not be the preserve of techno-determinists, but be open to all. For example, ubiquitous computing can be involved in many scenarios, not only that of "Ambient Intelligence". We need to engage in the development and critique of these different perspectives and approaches. Being able to work in and with multidisciplinary teams embodying distint, and at times conflicting perspectives, being able to communicate ones ideas and information across a variety of social and institutional boundaries, will become of great importance. Of particular concern, in the context of an IFIP INTERACT event, is the need to balance the heterogeneity of concepts and methods being used in research and practice with some form of quality control. Despite the heterogeneity of perspectives and disciplines nowadays involved in the field, I will argue that the HCI community, as a community, still does have a significant role to play in the development and evolution of useful, usable and enaging ICT-enabled infrastructures and applications. 